\title{
The Role of Activity BASED ACCOUNTING IN ATHLETIC DEPARTMENTS: THEORY, RESEARCH QUESTIONS, AND AN EMPIRICAL TEST
}

Jimmy Smith, Ian Burt, and Michael Gentile

The BRC Academy Journal of Business 9, no. 1 (2019): 1-32.

http://dx.doi.org/10.15239/j.brcacadjb.2019.09.01.ja01

Web APPENDIX

http://dx.doi.org/10.15239/j.brcacadjb.2019.09.01.wa01 
Table 1 Conference Representation

\begin{tabular}{|c|c|c|}
\hline Count & Percent & Conference \\
\hline & & American Athletic \\
\hline 1 & $2.50 \%$ & Conference \\
\hline 1 & $2.50 \%$ & Pac-12 Conference \\
\hline 1 & $2.50 \%$ & Big Ten Conference \\
\hline 2 & $5.00 \%$ & Mid-American Conference \\
\hline 1 & $2.50 \%$ & Sun Belt Conference \\
\hline 6 & $15.00 \%$ & Atlantic Coast Conference \\
\hline 4 & $10.00 \%$ & Mountain West Conference \\
\hline 1 & $2.50 \%$ & Conference USA \\
\hline 2 & $5.00 \%$ & Big 12 Conference \\
\hline \multirow[t]{2}{*}{1} & $2.50 \%$ & Southeastern Conference \\
\hline & & Southwestern Athletic \\
\hline 1 & $2.50 \%$ & Conference \\
\hline 0 & $0.00 \%$ & The Ivy League \\
\hline 2 & $5.00 \%$ & Big Sky Conference \\
\hline 4 & $10.00 \%$ & Atlantic 10 Conference \\
\hline 1 & $2.50 \%$ & Ohio Valley Conference \\
\hline
\end{tabular}




\begin{tabular}{|c|c|c|}
\hline 1 & $2.50 \%$ & Big East Conference \\
\hline 1 & $2.50 \%$ & Big South Conference \\
\hline 1 & $0.00 \%$ & Northeast Conference \\
\hline \multirow[t]{2}{*}{2} & $5.00 \%$ & Patriot League \\
\hline & & Mid-Eastern Athletic \\
\hline 1 & $2.50 \%$ & Conference \\
\hline 1 & $2.50 \%$ & Southland Conference \\
\hline \multirow[t]{2}{*}{2} & $5.00 \%$ & Atlantic Sun Conference \\
\hline & & Metro Atlantic Athletic \\
\hline 2 & $5.00 \%$ & Conference \\
\hline 2 & $5.00 \%$ & West Coast Conference \\
\hline 1 & $2.50 \%$ & Big West Conference \\
\hline 1 & $2.50 \%$ & Missouri Valley Conference \\
\hline 1 & $2.50 \%$ & American East Conference \\
\hline 1 & $2.50 \%$ & Horizon League \\
\hline 2 & $5.00 \%$ & Western Athletic Conference \\
\hline 1 & $2.50 \%$ & Colonial Athletic Association \\
\hline 2 & $5.00 \%$ & The Summit League \\
\hline 50 & $100 \%$ & \\
\hline
\end{tabular}


Table 2 Specific accounting technique/format used when creating reports

\begin{tabular}{|ccl|}
\hline Count & Percent & Method/Format \\
\hline 4 & $7.84 \%$ & Activity Based Costing \\
21 & $41.17 \%$ & GAAP \\
25 & $49.02 \%$ & Zero-based budgeting \\
1 & $1.96 \%$ & Other \\
\hline 51 & $100 \%$ & \\
\hline
\end{tabular}


Table 3 Allocation of indirect costs

Panel A: Do you allocate indirect costs on a per team basis when creating internal reports?

\begin{tabular}{|ccl|}
\hline Count & Percent & Response \\
\hline 5 & $10.00 \%$ & Yes \\
45 & $90.00 \%$ & No \\
\hline
\end{tabular}

Panel B: Do you allocate indirect costs on a per team basis when creating external reports (NCAA, EADA, or IRS regulatory reports)?

\begin{tabular}{|cll|}
\hline Count & Percent & Response \\
\hline 12 & $24.00 \%$ & Yes \\
38 & $76.00 \%$ & No \\
\hline
\end{tabular}


Panel C: Do departments allocate indirect costs to each team?

\begin{tabular}{|cll|}
\hline Count & Percent & Allocation Method \\
\hline 37 & $74.00 \%$ & We don't \\
4 & $8.00 \%$ & Based on usage \\
1 & $2.00 \%$ & Based on \# of athletes \\
0 & $0.00 \%$ & Equal $\%$ for each team \\
3 & & Based on total costs of \\
4 & $6.00 \%$ & team \\
& $8.00 \%$ & Square footage of facility \\
\hline 50 & $2.00 \%$ & Other \\
\hline
\end{tabular}

